\title{
WEB RADIO COMO FERRAMENTA DE DIÁLOGO EM SAÚDE COLETIVA NO SERTÃO: JUVENTUDES E MÉTODOS CONTRACEPTIVOS
}

\author{
WEB RADIO AS A TOOL FOR DIALOGUE IN COLLECTIVE HEALTH IN THE \\ BRAZILIAN SERTÃO: YOUTHS AND CONTRACEPTIVE METHODS
}

WEB RADIO COMO UNA HERRAMIENTA DE DIÁLOGO EN SALUD COLECTIVA EN EL SERTÃO BRASILEÑO: JUVENTUDES Y MÉTODOS ANTICONCEPTIVOS

Palavras-chave: Juventude; Saúde; Tecnologias.

Keywords: Youth; Health; Technologies.

Palabras clave: Juventud; Salud; Tecnologías.

Submetido: 23/11/2017

Aprovado: 02/05/2018

Autor(a) para Correspondência: Leidy Dayane Paiva de Abreu End: Av. Antônio Justa, Condomínio Edificio Bolzano, 3799 CEP: 60165-090, Varjota, Fortaleza-CE E-mail: dayannepaiva@hotmail.com

\section{RESUMO}

Este artigo descreve o uso de uma web radio como ferramenta de diálogo em saúde coletiva no sertão brasileiro, com enfoque nas juventudes $e$ nos métodos contraceptivos. Trata-se de pesquisa exploratória e descritiva realizada em agosto de 2016 em Hidrolândia-CE, no âmbito do projeto "Em Sintonia com a Saúde", com estudantes de uma escola estadual de educação profissional (EEEP) e com jovens do "Baú da Leitura", da Associação dos Jovens do Irajá (AJIr). Foram realizadas observações in loco e 3 sessões educativas. As "perguntas-discursos" foram extraídas das redes sociais e de áudios do mural de recados da web radio e a análise recorreu à categorização das falas. Este estudo foi aprovado pelo Comitê de Ética em Pesquisa da Universidade Estadual do Ceará (UECE), em conformidade com a Resolução n. 466/2012 do Conselho Nacional de Saúde (CNS), e sua discussão enfoca os métodos contraceptivos e as tecnologias da comunicação. Nesse contexto, o uso da web radio se mostrou de suma importância para identificar dúvidas, conhecimentos e práticas relativas à contracepção. A enfermagem se destaca como categoria do setor saúde responsável pela qualidade da assistência, com uso das tecnologias da comunicação no cenário da saúde coletiva a serviço das juventudes.

\footnotetext{
1. Enfermeira. Aluna no Programa de Pós-Graduação Cuidados Clínicos em Enfermagem e Saúde da Universidade Estadual do Ceará (UECE). Fortaleza (CE), Brasil. E-mail: dayannepaiva@hotmail.com

2. Enfermeiro. Professor no Curso de Graduação em Enfermagem e no Programa de Pós-Graduação Cuidados Clínicos em Enfermagem e Saúde da UECE. Fortaleza (CE),Brasil.E-mail: augustomtorres@gmail.com 3. Enfermeira. Professora no Curso de Graduação em Enfermagem e no Programa de Pós-Graduação Cuidados Clínicos em Enfermagem e Saúde da UECE. Fortaleza (CE), Brasil. E-mail: rocineideferreira@gmail.com 4. Enfermeira. Aluna no Programa de Pós-Graduação Cuidados Clínicos em Enfermagem e Saúde da UECE. Fortaleza (CE),Brasil.E-mail: aretha.feitosa@gmail.com
} 


\section{ABSTRACT}

This article describes the use of a web radio as a tool for dialogue in collective health in the Brazilian sertão, with a focus on youths and contraceptive methods. This is an exploratory and descriptive research conducted in August 2016 in Hidrolândia, Ceará, Brazil, within the project 'Em Sintonia com a Saúde,' with students from a Brazilian professional education state school (EEEP) and with young individuals from the 'Baú da Leitura,'of the Associação dos Jovens do Irajá (AJIr). On-site observations and 3 educational sessions were carried out. The 'questions-discussions' were extracted from the social networks and audios on the message board of the web radio and the analysis resorted to categorization of the speeches. This study has been approved by the Research Ethics Committee of the Ceará State University (UECE), according to Resolution no. 466/2012 of the Brazilian National Health Council (CNS), and its discussion focuses on contraceptive methods and communication technologies. In this context, using the web radio showed to be extremely significant to identify doubts, knowledge, and practices related to contraception. Nursing stands out as a category in the health sector responsible for the quality of care, with use of communication technologies in the scenario of collective health at the service of youths.

\section{RESUMEN}

Este artículo describe el uso de una web radio como una herramienta de diálogo en salud colectiva en el sertão brasileño, con enfoque en las juventudes y los métodos anticonceptivos. Esta es una investigación exploratoria y descriptiva realizada en agosto de 2016 en Hidrolândia, Ceará, Brasil, dentro del proyecto "Em Sintonia com a Saúde", con estudiantes de una escuela estatal de educación profesional brasileña (EEEP) y con jóvenes del "Baú da Leitura", de la Associação dos Jovens do Irajá (AJIr). Se realizaron observaciones in loco y 3 sesiones educativas. Las "preguntas-discusiones" se extrajeron de redes sociales y audios en el tablero de mensajes de la web radio y el análisis recurrió a la categorización de las hablas. Este estudio ha sido aprobado por el Comité de Ética en Investigación de la Universidad del Estado de Ceará (UECE), de acuerdo con la Resolución no. 466/2012 del Consejo Nacional de Salud brasileño (CNS), y su discusión se centra en métodos anticonceptivos y tecnologías de comunicación. En este contexto, el uso de la web radio demostró ser extremadamente significativo para identificar dudas, conocimientos y prácticas relacionadas con la anticoncepción. La enfermería se destaca como categoría en el sector salud responsable de la calidad de la atención, con uso de las tecnologías de comunicación en el escenario de la salud colectiva al servicio de las juventudes.

\section{INTRODUÇÃO}

Adolescentes e jovens adoecem menos que outros grupos etários e, de fato, apresentam taxas de morbidade e mortalidade mais baixas do que as da população em geral. Entretanto, se esse público for observado em detalhe, evidencia-se, além do aumento da gravidez precoce, o avanço das infecções sexualmente transmissíveis (IST) denominadas vírus da imunodeficiência humana (HIV) e síndrome da imunodeficiência adquirida (AIDS), que, associadas a fatores socioeconômicos e culturais, têm profundas repercussões na qualidade de vida dessa parcela da população ${ }^{1}$.

Percebe-se, no setor saúde, uma imprecisão conceitual de adolescência, jovens e juventude partindo dos limites etários. Dentre as diversas políticas de Estado relativas à adolescência há aquelas que levam em conta os limites estabelecidos pelo Estatuto da Criança e do Adolescente (ECA) - de 12 a 18 anos - ou pelo Ministério da Saúde - de 10 a
19 anos. Para os jovens e a juventude, o Conselho Nacional de Juventude (Conjuve) estabelece a faixa etária de 15 a 29 anos - e também há propostas de organizações internacionais que ora aumentam, ora diminuem os limites da adolescência e da juventude ${ }^{2}$.

Preferiu-se adotar os termos jovens e juventudes ao invés de adolescentes e adolescências, uma vez que podem não se referir estritamente a uma faixa etária específica ou a uma série de comportamentos reconhecidos biologicamente. A escolha do termo jovem decorre do fato desse público estar imerso em cenários culturais diversos, produzindo suas vidas mediadas pelos cotidianos de suas experimentações e vivências em grupos e em outros territórios.

A problemática da saúde sexual e reprodutiva, mais especificamente dos métodos contraceptivos, constitui um tema relevante a abordar com os jovens logo no início de sua vida sexual - que ocorre cada vez precocemente. Isso se deve ao fato de que muitos indivíduos são suscetíveis a HIV, AIDS e gravidez indesejada, o que pode trazer consequências como 
aborto e mortalidade materno-infantil, dentre outras.

Comumente, as abordagens educativas sobre saúde sexual e reprodutiva ficam restritas à informação sobre doenças. Nesse cenário, surgem as novas tecnologias de educação em saúde; a informática educativa oferece as chamadas tecnologias interativas como ferramentas do saber teórico-prático. 0s jovens têm incorporado o universo digital como canal para o diálogo em saúde dentro e fora da escola. Nada obstante, o profissional da saúde, em especial o enfermeiro, deve lançar mão de recursos que envolvam o público jovem nos serviços de saúde, como no caso das tecnologias digitais de informação e comunicação (TDIC), propondo aos jovens um novo foco sem os tirar de seu campo de interesses ${ }^{3}$.

Assim, as práticas de cuidados em saúde requerem a apropriação do uso de novas tecnologias do cuidar, compreendidas estas como práticas virtuais que possibilitam ampliar o poder de diálogo e comunicação entre os sujeitos envolvidos no processo de promoção da saúde 4 .

Essas tecnologias se materializam por diversas ferramentas virtuais de que a própria internet dispõe, como web TV, fóruns, blogs, chats, aplicativos de smartphone, entre outros. A grande vantagem de seu uso é a rapidez no compartilhamento de informações, que se dá em poucos segundos, com ampla abrangência (praticamente mundial). Assim, seu uso constitui ferramenta pedagógica eficaz que o enfermeiro pode usar no compartilhamento de saberes com as juventudes ${ }^{5}$.

0 uso de tecnologias de comunicação nos processos do cuidar cria possibilidades e gera atração, sobretudo quando se trata das juventudes, que as utilizam com maior frequência em seu cotidiano de vida ${ }^{6}$. Assim, esses espaços virtuais, quando utilizados nas práticas de educação em saúde e promoção da saúde, representam um avanço na saúde coletiva.

Como exemplo do uso das TDIC, pode-se destacar o uso de web radio, um ambiente virtual de promoção do cuidado de enfermagem com jovens nas escolas e nos mais variados cenários da vida, o qual constitui uma experiência do uso das tecnologias digitais no processo de comunicação e educação em saúde.

Esta pesquisa adotou a seguinte questão norteadora:

- De que modo o uso de uma web radio potencializa as práticas de cuidado em saúde coletiva junto aos jovens, pautando-se em estratégias voltadas à saúde sexual e reprodutiva, com ênfase no uso de métodos contraceptivos?

\section{Os jovens têm \\ incorporado o universo \\ digital como canal para \\ o diálogo em saúde \\ dentro e fora da escola.}

Assim, esta pesquisa descreve o uso de uma web radio como ferramenta de diálogo em saúde coletiva, com enfoque nos métodos contraceptivos junto às juventudes do sertão brasileiro.

\section{METODOLOGIA}

Trata-se de pesquisa exploratório-descritiva com abordagem qualitativa, realizada no ciberespaço da web radio da Associação dos Jovens do Irajá (AJIr) e no espaço real, por meio de atividade de extensão universitária realizada por monitores do projeto “Em Sintonia com a Saúde" - alunos de mestrado e doutorado do Programa de Pós-Graduação Cuidados Clínicos em Enfermagem e Saúde da Universidade Estadual do Ceará (UECE). Essa etapa ocorreu em agosto de 2016 em Hidrolândia-CE, com estudantes do 2 o ano da do Curso Técnico em Agronegócio da Escola Estadual de Educação Profissional (EEEP) Francisca Maura, com idades de 15 a 17 anos, e jovens do "Baú da Leitura", da AJIr, com idades de 10 a 19 anos.

Os ciberespaços podem ser definidos como ambientes de interação e comunicação entre as pessoas que, intermediados pela rede de computadores e internet, faz circular informações de natureza virtual e real. 0 s termos virtual e real, com o primeiro não sendo a ausência do segundo, põem em destaque tensões que implicam tal dualidade, ainda que não sustentem uma teoria geral?

Os programas são produzidos em estúdio na UECE, no âmbito do projeto "Em Sintonia com a Saúde", e transmitidos via internet ao vivo pela web radio, às quartas-feiras, das $16: 00$ às 17:00. As discussões englobam temas de saúde coletiva em sessões semanais de debate. Para falar sobre cada tema são convidados profissionais especialistas na área, que dialogam e interagem com os jovens.

Primeiro, realizou-se um levantamento da realidade e planejamento, com visita in loco, conversa prévia com as juventudes e uso da observação sistemática. Constatou-se que o grupo está inserido no cenário de 
palavras que envolvem a saúde sexual e reprodutiva, mais especificamente 0 uso dos métodos contraceptivos. Posteriormente, foram realizadas 2 sessões educativas sobre saúde sexual e métodos contraceptivos, desenvolvidas em 2 momentos diferentes. 0 primeiro momento para os jovens do "Baú da Leitura" e o segundo para juventudes da EEEP.

A interação de jovens estudantes e entrevistados, para elaboração de perguntas-discussões e esclarecimento de dúvidas, ocorreu por meio de um dos links adotados (<www.ajir.com.br> ou <www. uece.ajir.com.br>) e dos demais canais de comunicação: Twitter; Skype; Facebook; mural de recados do site, blog e WhatsApp.

Para coleta de dados foi usado material das entrevistas sobre métodos contraceptivos, por meio de perguntas-discursos que os participantes enviaram via web radio, durante as interações da produção do programa na sala-estúdio com as juventudes nos territórios. Adotou-se para análise a técnica de agrupamentos e categorização das falas ${ }^{8,9}$.

Para garantir o anonimato dos participantes, recorreu-se a codinomes: Jovem 1, Jovem 2 [...] Jovem 24. Os locais foram denominados Território A e Território B. Este estudo foi aprovado pelo Comitê de Ética em Pesquisa da UECE, sob o Parecer n. 1.761.115, em conformidade com a Resolução n. 466/2012 do Conselho Nacional de Saúde (CNS).

\section{RESULTADOS E DISCUSSÃO}

0s resultados foram divididos em 3 categorias: a) Levantamento da realidade - apreensão do real por meio da observação in loco e da interação dialógica produzida entre pesquisador, web radio e juventudes; b) Primeira sessão - dialogando com as juventudes sobre os métodos contraceptivos; e c) Segunda sessão - protagonismo dos jovens acerca dos métodos contraceptivos no cotidiano escolar.

$$
\begin{aligned}
& \text {..os jovens } \\
& \text { expressaram desejo } \\
& \text { de dialogar sobre } \\
& \text { saúde sexual e } \\
& \text { reprodutiva. }
\end{aligned}
$$

Levantamento da realidade - apreensão do real por meio da observação in loco e da interação dialógica produzida entre pesquisador, web radio e juventudes

Por meio das visitas in loco e conversas prévias com as juventudes dos 2 territórios (EEEP e AJIr), observou-se no decorrer das ações de extensão universitária e em conversas informais que os jovens expressaram desejo de dialogar sobre saúde sexual e reprodutiva.

Além disso, as juventudes se mostraram mais ativas e mais comunicativas e não se dispersaram durante a discussão dos temas saúde sexual e reprodutiva via web radio. A sessão sobre os métodos contraceptivos constituiu tema central das perguntasdiscussões, seguida pelo planejamento familiar, IST/ HIV/AIDS, identidade e gênero.

No decorrer das observações in loco, apreenderamse os termos mais frequentes no universo das juventudes: namoro, gravidez, IST/HIV/AIDS, camisinha, métodos contraceptivos, celulares, tablets, aplicativos, blogs, chats, Facebook, Twitter, WhatsApp, games e YouTube, entre outros.

Em uma das atividades do projeto de extensão universitária, realizada na EEEP com um grupo de 25 jovens do $2^{\circ}$ ano (15 do sexo feminino e 10 do sexo masculino), com idades de 15 a 17 anos, perguntouse:

- "Se fosse para construir uma tecnologia educativa, o que vocês construiriam?"

A maioria (21) respondeu:

- “Um jogo para celulares dinâmico e lúdico, com perguntas relacionadas a namoro, relação sexual e como se prevenir."

Descreveram-se impressões, conhecimentos e como os jovens desejavam a construção do software. Para isso foram realizadas 2 sessões, 1 em cada território, em dias diferentes. A primeira sessão foi realizada na AJIr e a segunda na EEEP, abordando o uso dos métodos contraceptivos, como se descreve a seguir.

\section{Primeira sessão - dialogando com as juventudes sobre os métodos contraceptivos}

Descreve-se, aqui, a participação de 11 jovens, com idades de 10 a 19 anos, que participaram do "Baú da Leitura". Analisaram-se as perguntasdiscursos dos jovens sobre métodos contraceptivos. Vale ressaltar que essa foi uma programação especial, 
já que as atividades do "Baú da Leitura" ocorrem na AJIr aos sábados e as atividades da web radio ocorrem às quartas-feiras. 0 programa foi produzido das 17:00 às 18:00.

Os jovens enviavam perguntas pelas redes sociais Facebook e WhatsApp para o debatedor. Essas perguntasdiscursos apresentam diversos sentidos, como linhas de força nas falas dos participantes sobre sexualidade e saúde reprodutiva em cada categoria discursiva.

Quadro 1 - Perguntas-discursos dos jovens do Território A

\section{Temática: métodos contraceptivos}

- O que são métodos contraceptivos? Dizem que a camisinha é um. (Jovem 1)

- Quais são os mais usados? Só conheço a camisinha masculina. (Jovem 8)

- Várias amigas já engravidaram sem querer. Eu queria saber o que devemos saber para prevenir a gravidez e qual é o melhor método. (Jovem 9)

- A camisinha é o método mais seguro? Porque me disseram que ela previne contra as doenças. (Jovem 5)

- Conheço e a maioria dos meus amigos só conhece a camisinha masculina e a pílula, que são as mais usadas. Quais são os outros? (Jovem 7)

- O que devemos fazer para nos prevenir da AIDS? (Jovem 10)

- É verdade que posso pegar AIDS se eu não me prevenir? (Jovem 2)

- Eu tenho uma amiga que ficou grávida porque usou o coito interrompido e outra que pegou uma doença do namorado. Eles são irresponsáveis. Fiquei preocupada. Quais são as doenças que pegamos se não nos prevenimos? Como sei se tenho uma? 0 que vou sentir? (Jovem 4)

- Tudo que sei eu aprendi com meus amigos, tenho vergonha de meus pais, professores e enfermeiros. Como é que falamos pros pais da gente sobre isso? (Jovem 6)

- O que é DIU? O diafragma é o DIU? É a gente que bota? E não machuca? (Jovem 3)

Fonte: Elaborado pelos autores.

0 Quadro 1 evidencia a participação ativa da maioria (10) dos jovens do "Baú da Leitura" na realização de perguntas-discursos; 3 jovens mencionaram conhecer a camisinha masculina e 2 citaram a pílula e a camisinha masculina como métodos mais utilizados no planejamento familiar. Relataram, ainda, que a camisinha masculina é o método mais seguro para evitar doenças e 1 participante mencionou não conhecer alguns métodos contraceptivos (como DIU e diafragma), além de dúvidas em relação ao uso deles.

Apesar dos jovens verbalizarem algum conhecimento sobre métodos contraceptivos, gravidez indesejada e IST/HIV/AIDS, observaram-se dúvidas, curiosidades e falhas nos conceitos citados, surgindo a necessidade de abordar esse tema com maior ênfase ${ }^{9}$.

Nas perguntas, o preservativo masculino assume papel de destaque dentre os métodos contraceptivos, observando-se desconhecimento acerca dos outros métodos. 0 preservativo masculino e a pílula anticoncepcional são amplamente conhecidos pela juventude no Brasil ${ }^{10}$.

A discussão sobre gravidez e IST/HIV/AIDS possibilitou identificar as principais necessidades a trabalhar com esse público - particularmente preocupado com esses problemas.

0 HIV e a AIDS se encontram entre os principais problemas contemporâneos de saúde pública, em especial entre os jovens, mais suscetíveis a tais agravos, com as mais diversas implicações para a sociedade, perpassando aspectos objetivos e subjetivos. Vale ressaltar que se convive com duas epidemias distintas, embora intimamente relacionadas ${ }^{11}$.

No momento da sessão, os participantes citaram que a falta de responsabilidade de muitos jovens e relataram experiências de amigas que tiveram gravidez não planejada. Mencionaram, ainda, a falta de diálogo com os pais, professores e profissionais da saúde (como os enfermeiros).

Dentre os fatores que influenciam o não uso de métodos anticoncepcionais ou seu uso incorreto temos a falta de diálogo e a esporadicidade e o não planejamento das relações sexuais. Esse fato pode ser explicado pelo desconhecimento acerca dos modos seguros de prevenir essas situações ou do uso correto dos métodos ${ }^{12}$.

A juventude tem iniciado precocemente sua vida sexual, o que aumenta a incidência de gravidez não planejada. 0 conjunto de transformações fisiológicas, culturais, sociais, emocionais e psicológicas, 
combinado à falta de orientação sexual e discussão adequada sobre o planejamento familiar por parte da escola e do núcleo familiar, concretiza esse fato ${ }^{13}$.

Em relação à comunicação, percebe-se que a juventude se sente mais à vontade ao conversar com amigos e, muitas vezes, diante da falta de diálogo em seu núcleo familiar, os amigos constituem sua fonte de informações mais acessivel. Na família, o diálogo ainda é frágil ou inexistente; na escola, o debate se mostra tímido e, quando ocorre, volta-se mais aos aspectos biológicos, reforçando a ideia da sexualidade ligada à reprodução. Tanto educadores como profissionais da saúde ainda mantêm posturas impregnadas de preconceitos e tabus ${ }^{14}$.

Observou-se que o tema abordado faz parte da realidade e do convívio dos jovens. 0 momento foi proveitoso no sentido de esclarecer dúvidas sobre os métodos contraceptivos, abrindo espaço para discussão e contribuindo para uma reflexão crítica. Daí provém a importância da discussão das juventudes por meio da web radio, um meio de informação que rompe barreiras e favorece a comunicação e o diálogo mais aberto acerca do tema, menos carregado de timidez ou de dificuldade para se expressar livremente.

Independente da participação familiar no processo educativo, a sexualidade vem sendo abertamente debatida em meios de comunicação como a televisão, o rádio e a internet e isso tem influenciado o comportamento dos jovens com um bombardeio de informações - em sua maioria distorcidas - sobre sexualidade. É necessário adotar um olhar mais cuidadoso em relação a quais redes de informação esse público vem acessando. Os pais e familiares devem conhecer os espaços de comunicação e informação que esse público adentra ${ }^{15}$.

Mostra-se fundamental combinar informação e comunicação a discussão e orientação, no sentido de proporcionar à juventude maior esclarecimento quanto a suas dúvidas, seus receios e seus tabus relativos ao tema para, assim, abrir espaço à busca de informações e ao interesse em prevenir possíveis agravos relacionados à falta de cuidados voltados à vida sexual e reprodutiva.

\section{Segunda sessão - protagonismo dos jovens acerca dos métodos contraceptivos no cotidiano escolar}

A segunda sessão foi realizada na EEEP, mais especificamente em 2 turmas do $2^{ }$ano do Curso Técnico de Agronegócio. Ao todo, estavam presentes 70 alunos e essa sessão possibilitou 0 compartilhamento de dúvidas, ideias, conhecimentos e opiniões sobre o tema em questão. Foram realizadas 13 perguntas-discursos e o codinome dos jovens teve a mesma sequência da primeira sessão, como se pode observa no Quadro 2.

Quadro 2 - Perguntas-discursos dos jovens do Território B

\section{Temática: métodos contraceptivos}

- Existe uma possibilidade da camisinha masculina causar uma reação alérgica ao látex? (Jovem 12)

- O DIU previne contra as DST? (Jovem 13)

- O DIU incomoda de alguma forma o homem na relação sexual? (Jovem 14)

- Já ouvi comentários de que o DIU pode causar câncer. É verdade? (Jovem 15)

- Por que o DIU não previne em 100\% a gravidez? (Jovem 16)

- O anticoncepcional regula a menstruação? (Jovem 17)

- Se uma pessoa esquecer de tomar os comprimidos, pode correr risco de engravidar? (Jovem 18)

- Quando se toma anticoncepcional por muito tempo, há possibilidade da mulher ter dificuldade para engravidar? (Jovem 19)

- Onde os jovens podem obter informações sobre o tema? (Jovem 20)

- O que podemos fazer nas escolas para passar o que aprendemos para os estudantes e a comunidade onde moramos? (Jovem 21)

- Porque o setor saúde não está tão presente nas escolas? (Jovem 22)

- Qual é a importância de tratar esse tema com os jovens nas escolas? (Jovem 23)

- Por que, apesar de termos tantas informações, existem muitos amigos meus que não têm conhecimento e pegam doenças como a AIDS e outras pessoas ficam grávidas? (Jovem 24)

Fonte: Elaborado pelos autores.

Algumas dúvidas apresentavam um olhar mais social, ressaltando a importância da comunicação voltada ao 
tema e exigindo estratégias para que as informações cheguem à escola e à comunidade.

Vale ressaltar que há outras dúvidas a esclarecer, como no caso da camisinha masculina, do incômodo de alguns métodos contraceptivos, da pílula anticoncepcional e do uso correto do DIU.

Como se pôde verificar por meio dos questionamentos, os jovens não usam 0 anticoncepcional adequadamente, por não acreditarem na possibilidade de gravidez ou de IST/HIV/AIDS. Em razão da baixa frequência das relações sexuais, os jovens se consideram em situação de segurança, acham difícil obter os contraceptivos e sentem incômodo ao usá-los, além de assumirem a premissa de que "isso não vai acontecer comigo"14.

Novas perguntas surgiram e outros pontos foram questionados, como as informações em tempo real repassadas à juventude via internet; apesar delas, muitos jovens ainda desconhecem o tema ou fazem mau uso dos métodos contraceptivos. A web radio é um meio eficiente de diálogo e constitui uma ferramenta de escuta, promovendo a saúde, esclarecendo dúvidas e prevenindo ou diminuindo riscos e vulnerabilidades, além de fortalecer vínculos.

Nas interações, os participantes mostraram interesse em discutir, por meio das TDIC, o tema na escola, via diálogo aberto que permita sua livre expressão para o esclarecimento de dúvidas. Mencionaram a necessidade de espaços para a escuta e o compartilhamento dessas informações nos diversos locais em que estão inseridos, além da criação de estratégias para a difusão das informações sobre os métodos contraceptivos, onde possam ser protagonistas das ações e estabelecer vínculos com os professores, proporcionando a educação em saúde na escola.

Um dos pontos fundamentais para a efetividade das ações de planejamento reprodutivo é o vínculo estabelecido com o parceiro, os familiares, os professores e os profissionais da saúde, com acesso a informações e técnicas corretas, que possibilitem ao indivíduo fazer escolhas conscientes a partir de sua própria realidade, com autonomia, melhorando suas condições de vida e saúde ${ }^{15,16}$.

Vale salientar que o planejamento familiar não deve enfocar apenas o uso ou não dos métodos contraceptivos; deve-se orientar, dialogar e debater com a juventude os riscos e as consequências de iniciar sua vida sexual sem proteção, alertando sobre os modos de prevenção e transmissão de IST/HIV/ AIDS e os sintomas de tais doenças. Deve-se

\section{A web radio é um meio eficiente de diálogo e constitui uma ferramenta de escuta, promovendo a saúde...}

implementar uma educação em saúde crítica e reflexiva para intervenções comprometidas com os princípios da democracia - que rejeita qualquer modo de discriminação e dominação e busca a inovação e renovação no setor saúde ${ }^{17}$.

Depreende-se das perguntas a preocupação dos jovens em aprofundar seus conhecimentos acerca do tema, além do importante papel de debate assumido pelos profissionais da saúde na escola.

As estratégias de ensino e as pedagogias inovadoras, conhecidas como metodologias ativas, corroboram a ideia de que a busca do saber em Enfermagem deve aproximar a prática assistencial à prática educacional, já que o enfermeiro utiliza o processo ensino-aprendizagem em suas ações de saúde coletiva e na promoção da saúde junto aos jovens ${ }^{18}$. Logo, o empoderamento das juventudes passam a interferir em seu processo saúde-doença potencializa-se por meio do autocuidado ${ }^{19}$.

É necessário trabalhar a saúde dos jovens abordando o processo de educação em saúde, com uso das diversas tecnologias (como as TDIC), trazendo interação que enseje uma vivência participativa com ênfase no diálogo - campo profícuo para a reflexãoação e a elaboração coletiva de propostas sistematizadas de educação emancipatória em saúde.

Os participantes demonstraram interesse em construir um software educativo (jogo) para dialogar e discutir a promoção da saúde sexual e reprodutiva, de modo a combinar a ideia de cidadania aos direitos humanos, considerando que as atividades nos territórios constituem modos de fortalecer as ações de promoção da saúde junto à comunidade, com a aproximação entre os serviços de saúde e os indivíduos. As intervenções de educação em saúde assumem grande valor para o empoderamento dos jovens, que são estimulados a participar de seu plano terapêutico e a desenvolver sua autonomia.

\section{CONCLUSÃO}

A liberdade de diálogo proporcionada pela web radio apresentou resultados satisfatórios, tendo em 
vista o universo de palavras com que os jovens dialogam. Termos e expressões voltadas à saúde sexual e reprodutiva e às tecnologias digitais auxiliaram, nessa etapa exploratória, o planejamento da construção de um jogo (nos moldes de um aplicativo para celulares) voltado aos métodos contraceptivos.

Assim, este artigo traz um novo olhar às práticas em saúde coletiva com uso de ferramentas tecnológicas específicas sobre os métodos contraceptivos, com abordagem amplificada, a partir de uma web radio, que considera as realidades, as necessidades e as potencialidades das juventudes no sertão brasileiro.

\section{CONTRIBUIÇÃO DOS AUTORES}

Leidy Dayane Paiva de Abreu e Raimundo
Augusto Martins Torres contribuíram com o
delineamento e a realização da pesquisa e a
estruturação e redação do manuscrito. Maria
Rocineide Ferreira da Silva e Aretha Feitosa de
Araújo contribuíram com a revisão crítica do
manuscrito.

\section{REFERÊNCIAS}

1. Martins MG, Santos GHN, Sousa MS, Costa JEFB, Simões VM. Associação de gravidez na adolescência e prematuridade. Rev Bras Ginecol obstet [serial on the internet]. 2011 [cited 2018 May 4];33(11):354-60. Available from: http://www.scielo.br/pdf/rbgo/v33n11/a06v33n11. pdf

2. Brasil. Parâmetros Curriculares Nacionais: pluralidade cultural e orientação sexual. 2. ed. Rio de Janeiro: DP \& A; 2014.

3. Oliveira Júnior JK, Silva MAD. As tecnologias de informação e comunicação como ferramenta complementar no ensino da histologia nos cursos de odontologia da região Norte. J Health Inform [serial on the internet]. 2014 [cited 2018 May 15];6(2):60-6. Available from: http://www.jhisbis.saude.ws/ojs-jhi/index.php/jhi-sbis/article/ view $/ 293 / 196$

4. Amante LN, Rossetto AP, Schneider DG. Sistematização da assistência de enfermagem em unidade de terapia intensiva sustentada pela teoria de Wanda Horta. Rev Esc Enferm USP [serial on the internet]. 2009 [cited 2018 May 15];43(1):5464. Available from: http://www.scielo.br/pdf/reeusp/ v43n1/07.pdf

5. Rangel SML, Lamego G, Gomes ALC. Alimentação saudável: acesso à informação via mapas de navegação na internet. Physis (Rio J) [serial on the internet]. 2012 [cited 2018 May 15];22(3):919-39. Available from: http://www.scielo. br/pdf/physis/v22n3/05.pdf

6. Lira C, Ferreira L, Costa MJ, Pinto MK, Fernandes S. Discutindo violência e saúde na web-rádio: uma proposta intervencionista. Revista Extendere [serial on the internet]. 2015 [cited 2018 May 15];2(2):64-72. Available from: file:///D:/1287-3403-1-PB.pdf
7. Lévy, P. 0 que é virtual? Rio de Janeiro: Ed. 34; 2012

8. Minayo MC. 0 desafio do conhecimento: pesquisa qualitativa em saúde. 14. ed. São Paulo: Hucitec; 2014.

9. Torres RAM, Freitas GHF, Vieira DVF, Sales LMS. Tecnologias digitais e educação em enfermagem: a utilização de uma web rádio como estratégia pedagógica. J Health Inform [serial on the internet] . 2012 [cited 2018 May 15];4(1):1526. Available from: file:///D:/248-916-1-PB.pdf

10. Martins LB, Costa-Paiva L, Osis MJ, Sousa MH, Pinto Neto AM, Tadini V. Conhecimento sobre métodos anticoncepcionais por estudantes adolescentes. Rev Saúde Pública [serial on the internet]. 2006 [cited $2017 \mathrm{Mar}$ 12]; 40(1):57-64. Available from: http://www.scielo.br/pdf/ rsp/v40n1/27116.pdf

11. Carvalho AM, Rodrigues CS, Medrado KS. Oficinas em sexualidade humana com adolescentes. Estud Psicol (Natal) [serial on the internet]. 2015 [cited 2018 May 15];10(3):4316. Available from: http://www.scielo.br/pdf/epsic/v10n3/ a 06 v10n3.pdf

12. Chiamenti C. Uso de tecnologias da informação e comunicação no ensino presencial em enfermagem. Rio Grande (RS): Universidade Federal do Rio Grande; 2012.

13. Arcanjo CM, Oliveira MIV, Bezerra MGA. Gravidez em adolescentes de uma unidade municipal de saúde em Fortaleza - Ceará. Esc Anna Nery Rev Enferm [serial on the internet]. 2007 [cited 2018 May 15];11(3):445-51. Available from: http://www.scielo.br/pdf/ean/v11n3/v11n3a08.pdf

14. Berlofi LM, Alkmin ELC, Barbieri M, Guazelli CAF, Araújo FF. Prevenção da reincidência de gravidez em adolescentes: efeitos de um Programa de Planejamento Familiar. Acta Paul Enferm [serial on the internet]. 2006 [cited 2018 May 15];9(2):196-200. Available from: http://www.scielo.br/ $\mathrm{pdf} / \mathrm{ape} / \mathrm{v} 19 \mathrm{n} 2 / \mathrm{a} 11 \mathrm{v} 19 \mathrm{n} 2 . \mathrm{pdf}$

15. Imeida ACP. Produção de vídeos em sala de aula: uma proposta de uso pedagógico de celulares e câmeras digitais. \#Tear: Revista de Educação, Ciência e Tecnologia [serial on the internet]. 2013 [cited 2018 May 15];2(1):1-13. Available from: file:///D:/1785-5293-1-PB.pdf

16. Abreu LDP, Mendonça GMM, Andrade AC, Oliveira GR Aurélio D0, Rocha FAA. Abordagem educativa utilizando os Círculos de Cultura de Paulo Freire: experiência de acadêmicos de enfermagem no "Grupo Adolescer". Adolesc Saúde [serial on the internet]. 2013 [cited 2018 May 15];10(4):66-70. Available from: file:///D:/v10n4a09.pdf

17. Freire P. Educação como prática da liberdade. 27. ed. Rio de Janeiro: Paz e Terra; 2013.

18. Gartner Inc. Market share: mobile communication devices by region and country, 3011 [document on the internet]. 2012 [cited 2018 May 15]. Available from: https://www.gartner.com/doc/1847315/market-sharemobile-communication-devices.

19. Andrade MLR, Brito MCC, Freitas CASL. Planejamento familiar: um recurso estratégico à maternidade responsável de adolescentes primíparas. Sanare (Sobral, 0nline) [seria on the internet]. 2013 [cited 2018 May 15];12(1):27-32. Available from: file:///D:/325-628-1-SM.pdf

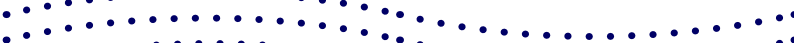

$\ldots \ldots \ldots \ldots$

$\ldots \ldots \ldots \ldots \ldots$

SANARE, Sobral - v.17, n.01,p.24-31, Jan./Jun. - 2018 - 31 